\title{
Proteomic analysis of susceptibility in intestinal stromal tumors
}

\author{
Y.C. Yang ${ }^{1}$, X. Cai ${ }^{2}$, S.Y. Zhang ${ }^{1}$, Z.L. Jia ${ }^{1}$, T.B. Chen ${ }^{1}$, L.R. Liu' ${ }^{1}$, J. Liu' ${ }^{1}$ and \\ M.C. Zhao' \\ ${ }^{1}$ Department of Clinical Laboratory, General Hospital of Beijing Military Area, \\ Beijing, China \\ ${ }^{2}$ Laboratory of Molecular Genetics, Beijing Institute of Basic Medical Sciences, \\ Beijing, China \\ Corresponding author: M.C. Zhao \\ E-mail: zhaomancangdcl@163.com
}

Genet. Mol. Res. 14 (4): 13566-13571 (2015)

Received April 9, 2015

Accepted July 23, 2015

Published October 28, 2015

DOI http://dx.doi.org/10.4238/2015.October.28.16

ABSTRACT. We analyzed the susceptibility of intestinal stromal tumors using cell culture and proteomics. Human SGC7901 gastric cells were selected and divided into a blank control group (untransfected SGC7901 cells), a negative control group [SGC7901 cells transfected with negative interference control-small interfering RNA (siRNA)], and a $\mathrm{COOH}$ terminus tensin-like molecule (CTEN)-siRNA-1 group (SGC7901 cells transfected with CTEN-siRNA-1). The cells were successfully transfected and subjected to analyses of cell proliferation, cell cycle, cell invasion, CTEN expression, and proteomics. The percentages of cells in the G0/ $\mathrm{G} 1, \mathrm{~S}$, and G2/M phases were similar in the three groups $(P>0.05)$, and the OD values were also similar at 24,48 , and $72 \mathrm{~h}(\mathrm{P}>0.05)$. Compared with the levels in the blank and negative control groups, CTEN protein in the CTEN-siRNA-1 group decreased by 66 and $65 \%$, respectively, and significantly fewer cells in the CTEN-siRNA-1 group were capable of invasion $(P<0.05)$. Proteomic analysis showed that in the CTEN-siRNA-1 group, 283 proteins were upregulated and 242 were downregulated; from these, the expression levels of E-cadherin and ERK proteins changed 
significantly. Silencing the expression of CTEN in intestinal stromal tumor cells reduces their invasion capability. Moreover, silencing CTEN at different stages can also regulate the expression levels of E-cadherin and ERK proteins.

Key words: Proteomics; Intestinal stromal tumor; Susceptibility; CTEN

\section{INTRODUCTION}

Gastric cancer is common in clinical practice, and gastrointestinal stromal tumor, a mesenchymal tumor of the gastrointestinal tract, has an annual morbidity rate of $10-20$ /million (Muler et al., 2002). It is often misdiagnosed as a smooth muscle- or nerve-derived tumor owing to the diverse tissue morphologies of residual smooth muscle tissues and nerve bundles (He et al., 2004). Because patients with gastric cancer, especially those in the medium and advanced stages, have poor prognosis, it is essential to unravel the molecular mechanisms behind the onset and progression of gastric cancer, and to seek novel intervention targets (Nishigaki et al., 2005). Tumor cells can maintain or change the internal and external environments in which they live, and proliferate by autocrine or paracrine mechanisms, and such factors have been highlighted in modern tumor molecular biology (Hong et al., 2013). Epithelial-mesenchymal transition (EMT), which refers to the transformation of fully differentiated epithelial cells into mesenchymal stem cells, plays an important role in normal embryonic development and tissue trauma repair, and mainly participates in organ fibrosis, tumor invasion, and metastasis (Ebert et al., 2005; Chen et al., 2013). As a member of the tensin gene family, COOH-terminus tensin-like molecule (CTEN) is involved in cell adhesion, migration, and signaling transduction. Moreover, it is highly expressed in many types of malignant tumors, being associated with the invasion and metastasis of tumors, as well as prognosis. However, gastric cancer-related EMT studies remain limited (Chen and Lo, 2003; Sandrasegaran et al., 2005).

Because proteomic analysis is currently spotlighted worldwide, we herein aimed to use it, together with cell culture, to explore the susceptibility of intestinal stromal tumors.

\section{MATERIAL AND METHODS}

\section{Cell source}

The human gastric cancer cell line SGC7901 was provided by the Department of Cell Biology, China Medical University. Two small interfering RNA (siRNA) primers were specifically designed according to the mRNA sequence of CTEN (NM 032865). The CTEN primers were designed by the Primer Premier 5 software according to the CTEN gene sequences.

\section{Cell culture}

Cells were cultured by conventional protocols and divided into a blank control group (untransfected SGC7901 cells), a negative control group (SGC7901 cells transfected with negative interference control-siRNA), and a CTEN-siRNA-1 group (SGC7901 cells transfected with CTEN-siRNA-1). 


\section{Western blotting}

An invasion assay was performed $24 \mathrm{~h}$ after successful transfection and proteins were collected $48 \mathrm{~h}$ after that for western blotting. CTEN expression was detected, and the cells were used for proliferation and cell cycle assays.

\section{Proteomic analysis}

Proteins from the cells were purified and analyzed by automatic 2D-Nano-LC-ESI-MS/MS equipment (20089832 type, ProtTech Inc., Suzhou, China). Peptide fragments were analyzed by strong-cation-exchange (SCX) reverse-phase nano-RPLC/ESI-MS/MS (03030706 type, Becton, Dickinson and Company, Franklin Lakes, NJ, USA).

\section{Statistical analysis}

Data were analyzed by SPSS 17.0. The numerical data were compared by chi-square test, and the categorical data were compared by $t$-test and univariate analysis of variance. $P<0.05$ was considered to be statistically significant.

\section{RESULTS}

\section{CTEN expression}

The transfection efficiency of siRNA was higher than $80 \%$, as observed under the microscope. Compared with the blank control group, two pairs of siRNA sequences inhibited the expression of CTEN. Compared with the blank and negative control groups, CTEN protein decreased by 66 and $65 \%$, respectively, in the CTEN-siRNA-1 group, indicating that the inhibitory system had been successfully established (Figure 1).

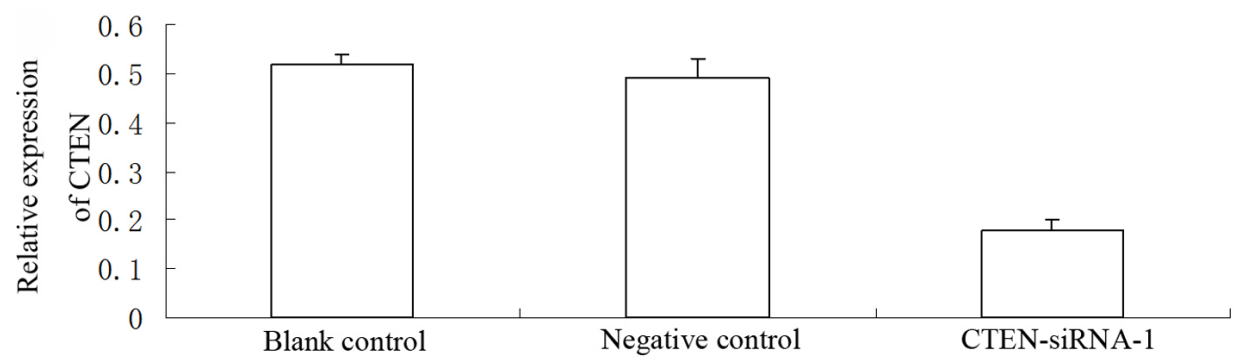

Figure 1. Effects of $\mathrm{COOH}$-terminus tensin-like molecule (CTEN) expression on cell proliferation in the three groups.

\section{Effects of CTEN on cell proliferation}

Cells in the three groups were inoculated onto 96-well plates, and their proliferation was detected using the CCK-8 method after 24,48 , and $72 \mathrm{~h}$ of transfection. The OD values were not statistically different at the three time points in the three groups $(P>0.05)$. Because transfection of CTEN-siRNA-1 did not noticeably affect the proliferation of SGC7901 cells, CTEN may have no effects (Table 1). 
Table 1. Effects of CTEN on the proliferative activity of SGC7901 cells (OD value, means \pm SD).

\begin{tabular}{|c|c|c|c|c|c|}
\hline Time (h) & Blank control group & Negative control group & CTEN-siRNA-1 group & $\mathrm{F}$ & $P$ \\
\hline 0 & $0.33 \pm 0.04$ & $0.32 \pm 0.07$ & $0.35 \pm 0.05$ & 0.453 & $>0.05$ \\
\hline 24 & $0.46 \pm 0.08$ & $0.43 \pm 0.12$ & $0.42 \pm 0.10$ & 0.619 & $>0.05$ \\
\hline 48 & $0.69 \pm 0.12$ & $0.62 \pm 0.11$ & $0.59 \pm 0.12$ & 0.554 & $>0.05$ \\
\hline 72 & $1.18 \pm 0.18$ & $1.05 \pm 0.15$ & $1.08 \pm 0.14$ & 0.399 & $>0.05$ \\
\hline
\end{tabular}

CTEN $=\mathrm{COOH}$-terminus tensin-like molecule; siRNA = small interfering RNA.

\section{Effects of CTEN on cell cycle}

Flow cytometry showed that the percentages of cells in the G0/G1, S, and G2/M phases were similar in the three groups $(P>0.05)$ after $48 \mathrm{~h}$ of transfection, suggesting that transfection of CTEN-siRNA-1 did not markedly affect the cell cycle in the SGC7901 cells (Table 2).

Table 2. Effects of CTEN on the cell cycle in SGC7901 cells (\%, means \pm SD).

\begin{tabular}{|c|c|c|c|c|c|}
\hline Phase & Blank control group & Negative control group & CTEN-siRNA-1 group & $\mathrm{F}$ & $P$ \\
\hline G0/G1 & $55.45 \pm 2.89$ & $54.34 \pm 1.23$ & $54.08 \pm 1.59$ & 0.412 & $>0.05$ \\
\hline $\mathrm{G} 2 / \mathrm{M}$ & $8.45 \pm 2.00$ & $8.07 \pm 0.78$ & $8.27 \pm 1.09$ & 0.554 & $>0.05$ \\
\hline $\mathrm{s}$ & $36.14 \pm 1.09$ & $37.62 \pm 1.13$ & $37.66 \pm 2.11$ & 0.310 & $>0.05$ \\
\hline
\end{tabular}

CTEN $=\mathrm{COOH}$-terminus tensin-like molecule; siRNA = small interfering RNA.

\section{Effects of CTEN on cell invasion}

A cell invasion assay was conducted using the small chamber method $24 \mathrm{~h}$ after transfection. Compared with the blank control $(44.87 \pm 2.98)$ and negative control $(43.37 \pm 3.98)$ groups, significantly fewer cells in the CTEN-siRNA-1 group $(22.78 \pm 4.20)$ were capable of invading $(P<0.05, F=18.390)$.

\section{Proteomic analysis}

Proteomic analysis showed that in the CTEN-siRNA-1 group, 283 proteins were upregulated and 242 were downregulated. The proteins were subjected to functional analysis (Figure 2). Proteins in the center, including E-cadherin and ERK, interacted with other proteins around them, which could be useful for further analysis.

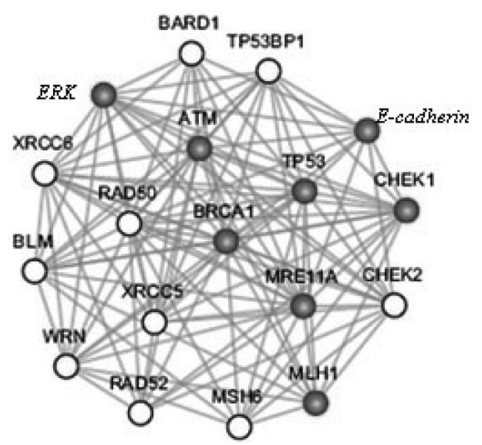

Figure 2. Proteomic analysis of the negative control and $\mathrm{COOH}$-terminus tensin-like molecule (CTEN)-small interfering RNA (siRNA)-1 groups. 


\section{DISCUSSION}

Gastric cancer, the most common malignant tumor of the digestive tract, endangers an increasing number of people in China annually. Despite some achievements, the prognosis is unsatisfactory and the mortality rate remains high. The onset and metastasis of tumors are closely associated with their external and internal microenvironments, which are subject to the structures, functions, and metabolism of the tissues involved (Albasri et al., 2009, 2011b). Independently originating from primitive mesenchymal tissues, gastrointestinal stromal tumors include smooth muscle cell-derived tumors, Schwann cell-derived tumors, and undifferentiated tumors that may have multiple differentiation capacities (Sakashita et al., 2008).

In patients with lung cancer and thymic carcinoma, the expression level of CTEN mRNA is positively correlated with tumor stage, and high expression is closely related to tumor stage and lymphatic metastasis (Kim et al., 2011; Lee et al., 2011). However, CTEN barely affected the proliferation of SGC7901 cells in this study, as evidenced by the slight influence of the transfecting CTEN-siRNA-1. Transfection with CTEN-siRNA-1 also exerted a negligible effect on the cells. The enhanced mobility and invasion capability of tumor cells are largely responsible for initiating the progression and metastasis of tumors. Epithelial tumor cells lose their intrinsic characteristics and begin to resemble mesenchymal stem cells during EMT, thereby acquiring migration and invasion capabilities (Albasri et al., 2011b). Overexpression of CTEN in intestinal cancer cells can decrease the expression levels of EMT key molecules and epithelial marker E-cadherin protein, as well as induce EMT by promoting cell migration and invasion. Therefore, CTEN may participate in EMT and facilitate invasion by inhibiting the expression of E-cadherin (Katz et al., 2007). In this study, compared with the blank and negative control groups, significantly fewer cells in the CTENsiRNA-1 group managed to invade $24 \mathrm{~h}$ after transfection $(P<0.05)$.

China has established a proteomic 2-DE database for human gastric tissues, providing crucial information for the proteomic analysis of normal tissues and those with pathological changes (Liao et al., 2009; Al-Ghamdi et al., 2013). Proteomic analysis revealed that 283 proteins were upregulated and 242 were downregulated in the CTEN-siRNA-1 group, of which the expression levels of E-cadherin and ERK proteins changed significantly. The expression of CTEN was probably augmented, and the expression of E-cadherin protein was thereby inhibited, which diminished intercellular adhesion and promoted EMT and invasion. ERK, as a serine/ threonine protein kinase and a member of the MAPK family, essentially regulates the onset, proliferation, and invasion of tumors. It has been reported that U0126, a specific inhibitor of the ERK pathway, can suppress epidermal growth factor-induced upregulation of CTEN expression by inhibiting the phosphorylation of ERK 1/2 kinase and blocking the ERK signaling pathway (Albasri et al., 2011a).

In summary, the susceptibility of intestinal stromal tumors is related to both individual genetic characteristics and interactions with the surrounding microenvironment. Silencing the expression of CTEN in gastric cancer cells can reduce their invasion capability. Moreover, silencing CTEN at different stages can also regulate the expression levels of E-cadherin and ERK proteins.

\section{Conflicts of interest}

The authors declare no conflict of interest. 


\section{REFERENCES}

Al-Ghamdi S, Cachat J, Albasri A, Ahmed M, et al. (2013). C-terminal tensin-like gene functions as an oncogene and promotes cell motility in pancreatic cancer. Pancreas 42: 135-140.

Albasri A, Seth R, Jackson D, Benhasouna A, et al. (2009). C-terminal tensin-like (CTEN) is an oncogene which alters cell motility possibly through repression of E-cadherin in colorectal cancer. J. Pathol. 218: 57-65.

Albasri A, Al-Ghamdi S, Fadhil W, Aleskandarany M, et al. (2011a). Cten signals through integrin-linked kinase (ILK) and may promote metastasis in colorectal cancer. Oncogene 30: 2997-3002.

Albasri A, Aleskandarany M, Benhasouna A, Powe DG, et al. (2011b). CTEN (C-terminal tensin-like), a novel oncogene overexpressed in invasive breast carcinoma of poor prognosis. Breast Cancer Res. Treat. 126: 47-54.

Chen $\mathrm{H}$ and Lo SH (2003). Regulation of tensin-promoted cell migration by its focal adhesion binding and Src homology domain 2. Biochem. J. 370: 1039-1045

Chen NT, Kuwabara Y, Conley C, Liao YC, et al. (2013). Phylogenetic analysis, expression patterns, and transcriptional regulation of human CTEN gene. Gene 520: 90-97.

Ebert MP, Krüger S, Fogeron ML, Lamer S, et al. (2005). Overexpression of cathepsin B in gastric cancer identified by proteome analysis. Proteomics 5: 1693-1704.

He QY, Cheung YH, Leung SY, Yuen ST, et al. (2004). Diverse proteomic alterations in gastric adenocarcinoma. Proteomics 4: 3276-3287.

Hong SY, Shih YP, Li T, Carraway KL 3rd, et al. (2013). CTEN prolongs signaling by EGFR through reducing its ligand-induced degradation. Cancer Res. 73: 5266-5276

Katz M, Amit I, Citri A, Shay T, et al. (2007). A reciprocal tensin-3-cten switch mediates EGF-driven mammary cell migration. Nat. Cell Biol. 9: 961-969.

Kim W, Bennett EJ, Huttlin EL, Guo A, et al. (2011). Systematic and quantitative assessment of the ubiquitin-modified proteome. Mol. Cell 44: 325-340.

Lee KA, Hammerle LP, Andrews PS, Stokes MP, et al. (2011). Ubiquitin ligase substrate identification through quantitative proteomics at both the protein and peptide levels. J. Biol. Chem. 286: 41530-41538.

Liao YC, Chen NT, Shih YP, Dong Y, et al. (2009). Up-regulation of C-terminal tensin-like molecule promotes the tumorigenicity of colon cancer through beta-catenin. Cancer Res. 69: 4563-4566.

Muler JH, Baker L and Zalupski MM (2002). Gastrointestinal stromal tumors: chemotherapy and imatinib. Curr. Oncol. Rep. 4: 499-503.

Nishigaki R, Osaki M, Hiratsuka M, Toda T, et al. (2005). Proteomic identification of differentially-expressed genes in human gastric carcinomas. Proteomics 5: 3205-3213.

Sakashita K, Mimori K, Tanaka F, Kamohara Y, et al. (2008). Prognostic relevance of Tensin4 expression in human gastric cancer. Ann. Surg. Oncol. 15: 2606-2613

Sandrasegaran K, Rajesh A, Rydberg J, Rushing DA, et al. (2005). Gastrointestinal stromal tumors: clinical, radiologic, and pathologic features. AJR Am. J. Roentgenol. 184: 803-811. 